\section{Macular hole in retinitis pigmentosa patients: microincision vitrectomy with polydimethylsiloxane as possible treatment}

EM Vingolo ${ }^{1}$, S Valente ${ }^{1}$, E Gerace ${ }^{1}$, L Spadea ${ }^{1}$ and $\mathrm{M}$ Nebbioso ${ }^{2}$

\section{Abstract}

Purpose To investigate long-term retinal changes after microincision pars plana vitrectomy surgery (MIVS) for macular hole (MH) in retinitis pigmentosa (RP) patientsretrospective and observational study. Methods Three RP patients suffering from MH were evaluated by means of best corrected visual acuity, anterior and posterior binocular examination, spectralis highresolution optical coherence tomography, MP-1 microperimetry (MP-1), and full-field electroretinogram (ERG), before MIVS and during the 36-month follow-up. Patients underwent simultaneous MIVS and microincision cataract surgery; IOL was positioned in capsular bag. Patients were hospitalised for 2 days after the surgery. Surgical procedure was performed according the following schedule: surgical removal of crystalline lens, MIVS with 23-gauge sutureless system trocars, core vitreous body removal, peeling of the inner limiting membrane, and balanced sterile saline solution-air-micro-structured polydimethylsiloxane (PDMS) exchange. PDMS tamponade, after 6 months starting from MIVS, was removed.

Results In all patients visual acuity increased after vitrectomy as a consequence of complete $\mathrm{MH}$ closure and restoration of retinal architecture. None of the patients developed ocular hypertension, or re-opening of $\mathrm{MH}$ during the 3-year follow-up. MP-1 bivariate contour ellipse area was reduced in its dimensions and improved in all patients demonstrating a better fixation.

Conclusions MIVS could be an effective treatment in RP patients with $\mathrm{MH}$ if medical therapy is not applicable or not sufficient. Finally more studies will be needed to improve knowledge about this genetic disease.

Eye (2015) 29, 699-702; doi:10.1038/eye.2014.344; published online 20 February 2015

\section{Introduction}

Retinitis pigmentosa (RP) includes a wide group of different degenerative diseases of the outer retina caused by gene abnormalities on several chromosomes and that leads to legal blindness in $1 / 4000 .{ }^{1}$ An estimated 1.5 million people are affected around the world. Some patients become blind as early as age 30; the majority of the patients are legally blind by age 60 with a central visual field diameter $<20$ degrees. ${ }^{1-3}$ The outer segment of photoreceptors are usually damaged in very early stage of the disease, while the macula appears spared from the degenerative process for a long time. ${ }^{2}$ Frequently the presence of liquid leakage from retinal capillaries ${ }^{4}$ causes cystoid macular oedema (CME) and/or cells migration in the vitreous body. ${ }^{5}$

Oishi et $a l^{6}$ classified the status of the inner segment/outer segment junction (IS/OS) as being absent, discontinuous, or distinct. As a consequence of chronic blood retinal barrier breakdown, which is reported to occur in $8-50 \%$ of the instances, the inner surface of the retina may present irregularities of the inner limiting membrane (ILM) or iperreflectivity of vitreoretinal interface. ${ }^{4,5}$ Macular cysts tend to be confluent in some cases and, in combination with ILM wrinkling, sometimes may lead to the formation of macular hole (MH) encompassing different stages (from I to IV according to Gass
${ }^{1}$ Department of

Ophthalmology, Polo Pontino, Policlinico Umberto I, Sapienza University of Rome, Rome, Italy

${ }^{2}$ Department of Sense Organs, Ocular Electrophysiology Center, Faculty of Medicine and Odontology, Policlinico Umberto I, Sapienza University of Rome, Rome, Italy

Correspondence: M Nebbioso, Department of Sense Organs, Ocular Electrophysiology Center, Faculty of Medicine and Odontology, Policlinico

Umberto I, Sapienza University of Rome, Piazzale Aldo Moro 5, Viale del Policlinico 155, Rome 00161, Italy

Tel: +39 06 49975422;

Fax: +3906 49975425 .

E-mail: marcella.nebbioso@ uniroma1.it

Received: 25 November 2013

Accepted in revised form: 22 December 2014 Published online: 20 February 2015 
classification), that in RP patients is present in $4-8 \%$ of eyes with different shapes and gradation. ${ }^{5}$ Consequently few reports of MH surgical treatment in RP patients are present in the literature and usually involve small number of eyes treated with pars plana vitrectomy. 7,8

The purpose of this study was to evaluate the morphological and functional outcomes of microincision pars plana vitrectomy surgery (MIVS) associated to ILM peeling technique in RP patients with $\mathrm{MH}$ when medical therapy is not applicable or not effective.

\section{Methods}

The clinical charts of $3 \mathrm{RP}$ patients suffering from $\mathrm{MH}$ secondary to chronic CME and tangential vitreoretinal tractions, who underwent 23-gauge MIVS (ACCURUS vitrectomy system ALCON Inc., Irvine, CA, USA) were evaluated retrospectively (Tables 1 and 2). These patients were unresponsive to conventional medical treatment based on carbonic anhydride inhibitors and nonsteroidal anti-inflammatory drugs or steroids.

Surgical procedure was performed with a three-port pars plana vitrectomy, peeling of the ILM, and simultaneous cataract surgery by the same surgeon (EMV) under retrobulbar anaesthesia (Table 3). Betamethasone $0.1 \%$ eye drops were instilled for 10 days, followed by fluorometholone $0.1 \%$ for several weeks. After 6 months starting from MIVS, polydimethylsiloxane (PDMS) was removed. Patients were followed up for 36 months.

\section{Patient G}

A 34-year-old male suffering from RP since the age of 14 . Baseline best corrected visual acuity (BCVA) was 6/20 Snellen in the right eye. Slit lamp examination only revealed a posterior subcapsular cataract. In addition, spectral-domain optical coherence tomography (SDOCT) demonstrated partial thickness MH and MP-1 reported a parafoveal fixation area with bivariate contour ellipse area (BCEA) of 0.42 degrees.

Due to the presence of $\mathrm{MH}$ (grade III/IV according to Gass classification), central retinal thickness was not considered, while parafoveal retinal thickness was $451 \mu \mathrm{m}$ (Figure 1a). After vitrectomy, BCVA increased to 10/20 Snellen. SD-OCT showed a considerable reduction in parafoveal retinal thickness, and demonstrated a central retinal thickness of $295 \mu \mathrm{m}$ (Figure 1b). BCEA decreased to a final value of 0.28 degrees.

\section{Patient A}

A 49-year-old male suffering from RP since the age 17. Baseline BCVA was 8/20 Snellen in the left eye. Nuclear and posterior subcapsular cataract was present at slit lamp examination. A partial thickness MH (grade II/III according to Gass classification) was demonstrated by

Table 1 Patients of the Referral Centre for Inherited Retinal Diseases

\begin{tabular}{|c|c|c|c|c|c|c|}
\hline Patients age & $\begin{array}{c}B C V A \\
\text { eye }\end{array}$ & Cataract & $\begin{array}{l}S D-O C T \\
\text { foveal }\end{array}$ & $\begin{array}{c}\text { Parafoveal } \\
\text { thickness }\end{array}$ & $\begin{array}{l}M P-1 \\
B C E A\end{array}$ & $\begin{array}{c}I O P \\
(m m H g)\end{array}$ \\
\hline \multicolumn{7}{|c|}{ Data before surgery treatment } \\
\hline G.O. (M) 34 yrs & 6/20 RE & Posterior subcapsular & MH III/IV & $451 \mu \mathrm{m}$ & 0.42 & 14 \\
\hline A.S. (M) 49 yrs & $8 / 20 \mathrm{LE}$ & Nuclear/posterior subcapsular & $\mathrm{MH}$ II/III & $350 \mu \mathrm{m}$ & 0.54 & 12 \\
\hline T.G. (F) 37 yrs & $2 / 20 \mathrm{RE}$ & Posterior subcapsular & MH IV & $440 \mu \mathrm{m}$ & 1.34 & 14 \\
\hline \multicolumn{7}{|c|}{ Data after vitrectomy: MICS/MIVS/PDMS } \\
\hline G.O. & $10 / 20$ & Phaco + IOL & $295 \mu \mathrm{m}$ & $350 \mu \mathrm{m}$ & 0.28 & 17 \\
\hline A.S. & $14 / 20$ & Phaco + IOL & $278 \mu \mathrm{m}$ & $295 \mu \mathrm{m}$ & 0.25 & 16 \\
\hline T.G. & $4 / 20$ & $\mathrm{Phaco}+\mathrm{IOL}$ & $290 \mu \mathrm{m}$ & $350 \mu \mathrm{m}$ & 0.98 & 17 \\
\hline
\end{tabular}

Abbreviations: BCEA, bivariate contour ellipse area in degrees (parafoveal fixation area); BCVA, best corrected visual acuity; F, female; IOP, intraocular pressure; LE, left eye; $\mathrm{M}$, male; $\mathrm{MH}$, macular hole (grade according to Gass classification); MICS, microincision cataract surgery; MIVS, microincision pars plana vitrectomy surgery; MP-1, microperimetry; PDMS, polydimethylsiloxane; Phaco + IOL, phacoemulsification with intraocular lens; RE, right eye; SD-OCT, spectral-domain optical coherence tomography (central and paracentral retinal thickness); T.G., patient with diagnosis of Usher syndrome.

Table 2 Exams performed before and after vitrectomy

\begin{tabular}{|c|c|c|c|c|}
\hline Exams performed & & & & \\
\hline $\begin{array}{l}\text { BCVA Snellen } \\
\text { and Bailey- } \\
\text { Lowson EDTRS } \\
\text { charts }\end{array}$ & $\begin{array}{l}\text { Anterior and } \\
\text { posterior } \\
\text { binocular } \\
\text { examination }\end{array}$ & $\begin{array}{l}\text { SD-OCT HRA (Heidelberg } \\
\text { Engineering, Heidelberg, Germany) } \\
\text { and Cirrus HD-OCT (Zeiss } \\
\text { Meditec, Inc., Dublin, CA, USA) }\end{array}$ & $\begin{array}{l}\text { Full-field ERG Stimulator MV } \\
\text { Monitor Mon-Pack } 120 \text { Metrovision } \\
\text { (Metrovision, Pérenchies, France) }\end{array}$ & $\begin{array}{l}\text { MP-1 microperimetry } \\
\text { (Nidek Technologies, } \\
\text { Padua, Italy (I)) }\end{array}$ \\
\hline
\end{tabular}

Abbreviations: BCVA, best corrected visual acuity; ERG, electroretinogram; SD-OCT, spectral-domain optical coherence tomography. 
SD-OCT. MP-1 reported a parafoveal fixation area with BCEA of 0.54 degrees at baseline. The parafoveal retina measured $350 \mu \mathrm{m}$ in thickness. Postoperative VA increased to $14 / 20$ Snellen. Central retinal thickness was $278 \mu \mathrm{m}$. BCEA after $\mathrm{MH}$ resolution was of 0.25 degrees.

\section{Patient T}

A 37-year-old woman with diagnosis of Usher syndrome. Baseline BCVA was 2/20 Snellen in the right eye.

Posterior subcapsular cataract was observed biomicroscopically. SD-OCT showed a full thickness $\mathrm{MH}$. A parafoveal fixation area with BCEA of 1.34 degrees at baseline was demonstrated with MP-1. Parafoveal retinal thickness was $440 \mu \mathrm{m}$. Postoperatively, VA increased to 4/20 Snellen. SD-OCT showed a reduction in parafoveal retinal thickness, and a central retinal thickness of $290 \mu \mathrm{m}$. BCEA decreased to 0.98 degrees.

\section{Discussion}

$\mathrm{MH}$ in $\mathrm{RP}$ patients is caused by chronic $\mathrm{CME}$, incomplete vitreous detachment, and traction of condensed vitreous

Table 3 Surgical procedure performed with a three-port pars plana vitrectomy under retrobulbar anaesthesia by the same surgeon (EMV), according to the following schedule and guidelines (the Declaration of Helsinki, Institutional Review Board, and informed consent was obtained from all subjects before enrolment)

\section{Surgical procedure}

Step 1 Surgical removal of the crystalline lens with MICS

Step 2 Phacoemulsification, aspiration, and IOL implantation

Step 3 Sclerotomy and MIVS with 23-gauge sutureless system trocars

Step 4 Core vitreous body removal with high aspiration level

Step 5 Posterior hyaloid and ILM peeling (disposable Eckardt forceps)

Step 6 BSS-air-micro-structured PDMS exchange in eyes with MH

Abbreviations: BSS, balanced sterile saline solution; ILM, inner limiting membrane; IOL, intraocular lens; MICS, microincision cataract surgery; MIVS, microincision pars plana vitrectomy surgery; PDMS, polydimethylsiloxane. strands. ${ }^{7,8}$ In exceptional cases, spontaneous resolution of a full thickness MH may occur. 8,9

Complete $\mathrm{MH}$ closure and restoration of retinal architecture in our patients lead to increased VA postoperatively and reduced BCEA with better fixation (Figure 1a and b).

Likewise an interesting study of Mahmoud et al ${ }^{10}$ showed that a combined lensectomy and vitrectomy procedure in P347L transgenic pigs was associated with retention of a significantly greater number of outer nuclear layer nuclei than in unoperated fellow eyes. Authors stated that consistent with the results obtained from other studies, the preservation of photoreceptors after surgery may be explained by the action of some diffusible substances or growth factors originating from the stimulated iris, ciliary body, and the retina. ${ }^{11}$ They could be: basic fibroblast growth factor (bFGF), neurotrophic growth factor (CNTF), brain-derived neurotrophic factor (BDNF), nerve growth factor (NGF), and lens-epithelium-derived growth factor (LEGF). ${ }^{11-13}$

Moreover, Mahomoud et al ${ }^{10}$ and Schori et al ${ }^{14}$ showed that an autoimmune response by lymphocytes and cytokines may be involved in the rescue process of retinal cells degeneration 8 weeks after surgery.

On the other hand, the development of narrow-gauge transconjunctival vitrectomy systems has improved the visual recovery following surgery. We think that, at this stage in our knowledge, removal of any vitreomacular traction with peeling of the ILM is probably the main reason for visual improvement and better fixation postoperatively. Recently Ocriplasmin, by degrading laminin and fibronectin at the vitreoretinal interface, may allow induction of posterior vitreous detachment in a non-invasive manner.

Furthermore, additional research is still needed for the greater understanding of the pathophysiology underlying the development of vitreomacular traction and idiopathic $\mathrm{MH}$ in RP patients too. ${ }^{15,16}$ Even if the present report is on few patients, our limited experience
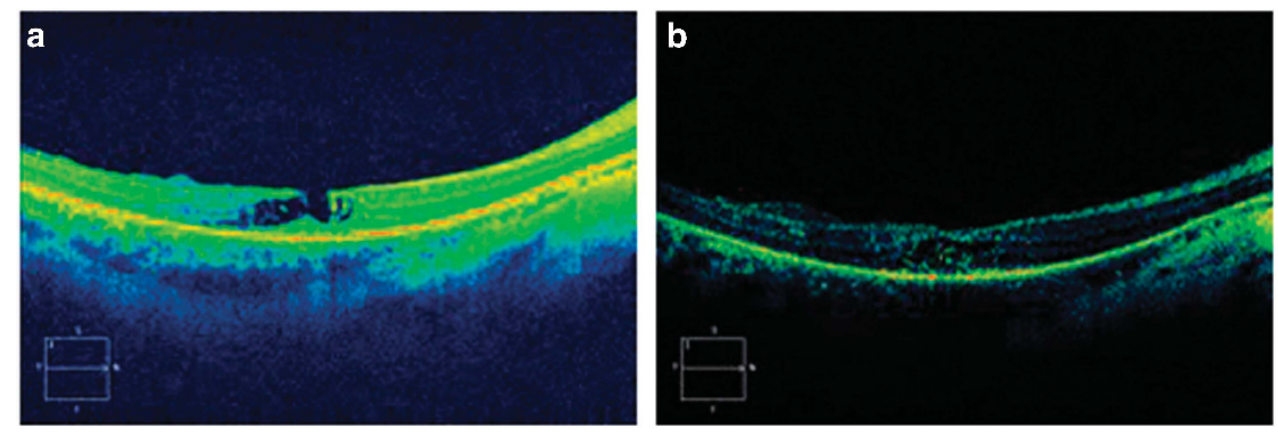

Figure 1 Retinitis pigmentosa (RP) in G.O. patient. (a) Macular hole (MH) grade III/IV, according to Gass classification, as shown by spectral-domain optical coherence tomography (SD-OCT) before surgery. Parafoveal retinal thickness was $451 \mu \mathrm{m}$. (b) After surgery, the MH closed and the central retinal thickness was $295 \mu \mathrm{m}$. 
suggests that MIVS could be an effective therapy for MH in tapetoretinal dystrophies, and according to Sandeep et al ${ }^{8,17-20}$ PDMS could be useful compared with air-gas tamponade since RP patients have visual field restriction that can cause movement and posture problems thus facilitating a recurrence of the disease. However, further evidence may be needed to confirm our preliminary findings.

\section{Summary}

What was known before

- RP includes degenerative diseases of the outer retina that leads to legal blindness

- Frequently the presence of liquid leakage from retinal capillaries causes CME

- Macular cysts tend to be confluent in some cases and may lead to the formation of $\mathrm{MH}$

\section{What this study adds}

- To evaluate the outcomes of MIVS associated to the inner limiting membrane peeling technique in retinitis pigmentosa patients with $\mathrm{MH}$ when medical therapy is not applicable or not effective

- Complete MH closure and restoration of retinal architecture in our patients lead to increased VA postoperatively

- The preservation of photoreceptors after surgery may be explained by the action of some diffusible substances or growth factors originating from the stimulated iris, ciliary body, and the retina

- Our limited experience suggests that MIVS could be an effective treatment for $\mathrm{MH}$ in tapetoretinal dystrophies

\section{Conflict of interest}

The authors declare no conflict of interest.

\section{References}

1 Berson EL. Retinitis pigmentosa. The Friedenwald lecture. Invest Ophthalmol Vis Sci 1993; 34(5): 1659-1676.

2 Grover S, Fishman GA, Anderson RJ, Alexander KR, Derlacki DJ. Rate of visual field loss in retinitis pigmentosa. Ophthalmology 1997; 104: 460-465.

3 Vingolo EM, Salvatore S, Belcaro G, Nebbioso M. Reduced retinal sensitivity and stereopsis in retinitis pigmentosa. Panminerva Med 2014; 56(S2): 29-32.

4 Hajali M, Fishman GA, Anderson RJ. The prevalence of cystoid macular oedema in retinitis pigmentosa patients determined by optical coherence tomography. $\mathrm{Br} \mathrm{J}$ Ophthalmol 2008; 92: 1065-1068.

5 Takezawa $\mathrm{M}$, Tetsuka A. Tangential vitreous traction: a possible mechanism of development of cystoids macular edema in retinitis pigmentosa. Clin Ophthalmol 2011; 5: 245-248.
6 Oishi A, Otani A, Sasahara M, Kojima H, Nakamura H, Kurimoto $\mathrm{M}$ et al. Photoreceptor integrity and visual acuity in cystoid macular oedema associated with retinitis pigmentosa. Eye (Lond) 2009; 23: 1411-1416.

7 Hagiwara A, Yamamoto S, Ogata K, Sugawara T, Hiramatsu A, Shibata $\mathrm{M}$ et al. Macular abnormalities in patients with retinitis pigmentosa: prevalence on OCT examination and outcomes of vitreoretinal surgery. Acta Ophthalmol 2011; 89: e122-e125.

8 Jin ZB, Gan DK, Xu GZ, Nao-I N. Macular hole formation in patients with retinitis pigmentosa and prognosis of pars plana vitrectomy. Retina 2008; 28: 610-614.

9 Raja M, Goldsmith C, Burton BJ. Spontaneous resolution of full-thickness macular hole in retinitis pigmentosa. BMJ Case Rep 2011; 2011: bcr0320113999.

10 Mahomoud TH, McCuen 2nd, BW, Hao Y, Moon SJ, Tatebayashi M, Stinnett $S$ et al. Lensectomy and vitrectomy decrease the rate of photoreceptor loss in rhodopsin P347L transgenic pigs. Graefes Arch Clin Exp Ophthalmol 2003; 241: 298-308.

11 Cao W, Li F, Steinberg RH, Lavail MM. Development of normal and injury-induced gene expression of aFGF, bFGF, CNTF, BDNF, GFAP and IGF-I in the rat retina. Exp Eye Res 2001; 72(5): 591-604.

12 Rush R. Immunohistochemical localization of endogenous nerve growth factor. Nature 1984; 312(5992): 364-367.

13 Machida S, Chaudry P, Shinobara T, Singh DP, Reddy VN, Chylack Jr, LT et al. Lens-epithelium-derived growth factor promotes photoreceptor survival in light-damaged and RCS rats. Invest Ophthalmol Vis Sci 2001; 42(5): 1087-1095.

14 Schori H, Kipnis J, Yoles E, WoldMussie E, Ruiz G, Wheeler LA et al. Vaccination for protection of retinal ganglion cells against death from glutamate cytotoxicity and ocular hypertension: Implications for glaucoma. Proc Natl Acad Sci USA 2001; 98(6): 3398-3403.

15 Steel DHW, Lotery AJ. Idiopathic vitreomacular traction and macular hole: a comprehensive review of pathophysiology, diagnosis, and treatment. Eye 2013; 27: S1-S21.

16 Malagola R, Iozzo N, Grenga P, Arrico L, Nebbioso M. Partial posterior vitreous detachment: new capabilities of improving diagnosis and surgical approach using SD-OCT. Ophthalmologica 2014; 232: 1-98.

17 Sandeep S, Carsten MH, Masahito O, Levent A. Vitreoretinal Surgery. JAYPEE highlights medical publishers: New Delhi, India, 2012, p 69.

18 Turano K, Herdman SJ, Dagnelie G. Visual stabilization of posture in retinitis pigmentosa and in artificially restricted visual fields. Invest Ophthalmol Vis Sci 1993; 34: 3004-3010.

19 Vingolo EM, Nebbioso M, Domanico D, Valente S, Frati P. Post-operative hospitalization in retinal detachment correlation to recurrences. Ann Ist Super Sanita 2013; 49(4): 336-339.

20 Vingolo EM, Gerace E, Valente S, Spadea L, Nebbioso M. Microincision vitrectomy surgery in vitreomacular traction syndrome of retinitis pigmentosa patients. Biomed Res Int 2014; 2014: 537081. 